\title{
UNIVERSIDAD NACIONAL Y COMPETITIVIDAD GLOBAL
}

\author{
NATIONAL AND GLOBAL COMPETITIVENESS UNIVERSITY \\ Raúl A. Arrarte Mera* \\ Docente Asociado de la Facultad de Ciencias Contables - UNMSM \\ [Recepción: Agosto de 2011 / Conformidad: Setiembre de 2011]
}

\section{RESUMEN}

Tres noticias de coyuntura propagadas en los medios sociales, en un lapso de 45 días, generaron nuestra inquietud para analizar el tema del rubro:

a) Creación de la Comisión de Reestructuración Administrativa, Orgánica y Funcional de la UNMSM, refrendada mediante Resolución Rectoral No 03377-R-11. Su instalación e inicio de actividades se produjo el 5 de agosto de 2011, en la Sala de Consejo, en el edificio Jorge Basadre. ${ }^{1}$.

b) Publicación de los "QS World University Rankings 2011/2012”" que se elaboró sobre la base de encuestas realizadas a académicos y empleadores, que evalúan la reputación de cada centro, basada en investigaciones realizadas. Cuyos 10 primeros puestos fueron ocupados por universidades británicas y estadounidenses.

c) El Proyecto de Ley del Grupo Parlamentario Nacionalista "Gana Perú”, que propone una nueva Ley Orgánica Universitaria.

Nuestro objetivo central es ofrecer información comparada y reflexionar acerca de las universidades del primer mundo respecto a sus pares de América Latina mejor posicionadas, para conocimiento de nuestras autoridades que buscan caminos de mejora competitiva para nuestra Alma Mater frente a los probables cambios institucionales a futuro en la Universidad Pública Peruana.

Palabras Clave: Competitividad Universitaria. Fortalezas de universidades británicas. Posicionamiento de universidades norteamericanas. Universidades Latinoamericanas. Universidades Peruanas.

\section{ABSTRACT}

Three situation news that spread in social media in a period of 45 days led to our concern to discuss said issue:

Creation of the Administrative Restructuring, Organic and Functional UNMSM Commission, countersigned through ViceChancellor Resolution No. 03377-R-11. The installation and start-up set was due on August 5, 2011, at the Council Chamber, Jorge Basadre Building. Publication of "QS World University Rankings 2011/2012" which was based on surveys to academics and employers, which evaluates the reputation of each College, based on ongoing research. 10 first positions were granted to British and American Universities. Nationalist Congress Group “Gana Peru” Bill which proposes a new University Act.

Our main objective is to provide comparative information and reflect on first world Universities in comparison to their best positioned Latin America counterparts, which provides information to our authorities who seek ways to improve our Alma Mater competitiveness to face possible future institutional changes at Peruvian State University.

Keywords: Competitiveness University. Strengths of British universities. Positioning of American universities. Latin American universities. Peruvian universities.

* Doctor en Ciencias Contables y Empresariales. Maestro en Administración. Contador Público Colegiado. E-mail: raularrarte@speedy.com.pe

1 Revista "San Marcos Al Día”. No. 327 de fecha 22/8/2011 a 23/9/2011. Página 9. UNMSM.

2 http://www.topuniversities.com/university-rankings/world-university-rankings/2011 consultado 15.09.2011 


\section{INTRODUCCIÓN}

En los últimos tiempos, Europa ha realizado importantes esfuerzos para aprovechar al máximo la investigación realizada en las universidades con fondos públicos y trasladarla a la sociedad y la empresa. De hecho, este continente se encuentra a la vanguardia de muchos campos científicos y técnicos. De momento, no se han obtenido los resultados esperados por falta de una red de cooperación. Por eso, la Unión Europea se ha propuesto crear un mercado común de la ciencia, semejante al que existe para bienes y servicios. Pretende que, en 2011, un 3\% del PBI (Producto Bruto Interno) se destine a la partida de investigación. Quizás, de esta manera consiga competir con países que actualmente son punteros en ciencia y tecnología como Estados Unidos y Japón.

Los investigadores que desarrollaron estos temas: María Ángeles Montoro, profesora de la Universidad Complutense de Madrid, y Eva María Mora, profesora de la Universidad Rey Juan Carlos, (España), afirman, en un trabajo publicado en Universia-Business Review, que existen dos grandes bloques de barreras y trabas a los que la sociedad universidad-empresa tiene que enfrentar: los problemas culturales y de comunicación, que son el origen de la mayoría de los obstáculos que surgen en este tipo de cooperación, y los problemas asociados a la obtención y explotación de los resultados de la investigación.

En concreto, el estudio explica que la universidad y la empresa cuentan con códigos éticos distintos. Así, mientras la primera basa su comportamiento en conductas como "la publicidad de los conocimientos generados a través de una actividad científica, la libertad de publicar el resultado de la investigación, el prestigio profesional, la calidad de las investigaciones y la generación de conocimientos", la segunda se rige por unas reglas prácticamente opuestas, como son "la privacidad de los conocimientos obtenidos en una investigación, la no-publicación de los resultados generados, el ánimo de lucro, la aplicación de las investigaciones a la estrategia de negocio y la mejora de su posición competitiva”.

Esta dicotomía nos inspiró a investigar cómo manejan esta situación los países ricos. Lo ideal es encontrar el punto medio para que todos ganen: universidad y empresa. Pero nuestro objetivo central es ofrecer información comparada y reflexionar acerca de las universidades del primer mundo respecto a sus pares de América Latina mejor posicionadas, para conocimiento de nuestras autoridades que buscan caminos de mejora competitiva para nuestra Alma Mater frente a los probables cambios institucionales a futuro en la Universidad Pública Peruana.

Analicemos la noticia que viene del exterior. Cambridge y Harvard, lideraron la encuesta. Y "entre los países latinoamericanos que figuran en la lista se encuentran México, (Universidad Nacional Autónoma de México), Brasil (Universidad de Sao Paulo), Chile (Pontificia Universidad Católica de Chile y Universidad de Chile) y Argentina (Universidad de Buenos Aires), en los puestos 169, $169,250,262$, y 270 , respectivamente". ${ }^{3}$

Lo que sigue es el resultado del análisis que encontramos en cada una de las páginas web de las instituciones universitarias analizadas.

3 Diario “Gestión”. Universidad de Cambridge es la más prestigiosa en el mundo. Lima. Perú. Página 30. Fechada 06.09.2011. 


\section{FORTALEZA DE UNIVERSIDADES BRITÁNICAS}

Inglaterra fue la cuna de la Revolución Industrial originaria de la economía manufacturera en la etapa de desarrollo del capitalismo de libre concurrencia o pre monopolista. A partir de la más rápida utilización de sus fuentes energéticas, de la mecanización de la industria textil, de la concentración industrial y de la hegemonía que ya poseía sobre las restantes metrópolis en la práctica de la colonización, esta nación llegó a ser líder indiscutible del capitalismo industrial a finales del siglo XVIII y durante el siglo XIX. Debido a esta acumulación de condiciones fue posible una cierta efervescencia de educación técnica en distintas ciudades inglesas, cuyo caso más destacado fue Manchester. ${ }^{4}$

En Oxford (fundado en 1167) y Cambridge (instituida en 1209) están los orígenes de la Universidad Inglesa de orden privado. La temprana fundación de las mismas les otorga el calificativo de universidades medievales en el lenguaje académico internacional.

Alberga una población estudiantil de diecisiete mil escolares que nutren las aulas de su Universidad, de los que cinco mil ochocientos son graduados. El once por ciento de la población estudiantil es extranjera, procedente de todas las partes del mundo. ${ }^{5}$

La Universidad de Cambridge está formada por un conglomerado de 35 colegios universitarios, cada uno con su propia organización, muy independientes, con gobierno, bienes e ingresos propios, si bien desde las últimas décadas están muy subvencionados con ayudas públicas. Responden de la administración rigurosa de sus alumnos y la selección de sus profesores, su acomodación y bienestar, con firme adhesión a las viejas tradiciones medievales, y contacto permanente de alumno con profesor, fuente de la envidiable vitalidad intelectual, espíritu crítico e investigación permanente, que ha producido más de 80 premios Nobel en el siglo $\mathrm{XX}{ }^{6}$

El personal de los "colleges" - fundaciones con personalidad jurídica propia -, está compuesto de "principal" (rector, decano, director), "tutor" (profesor dedicado intensamente a la enseñanza y formación de un número reducido de alumnos), "lecturers" o "readers" (profesores no permanentes, sin "tenure") y "undergraduates" (alumnos aún no graduados). Los graduados realizan estudios conducentes a cualquiera de los numerosos títulos de "master" existentes o de doctor. Los investigadores realizan su trabajo en los grandes centros de investigación, bien financiados y equipados por fundaciones nacionales o internacionales...?

¿Cómo se impartía la enseñanza? ¿Cuáles eran los métodos que han prevalecido con el paso del tiempo? Hay que distinguir entre las diversas áreas temáticas. La enseñanza de filosofía, que se fue extendiendo a las cátedras de historia, teología ("divinity"), derecho, medicina y matemáticas, incluía el estudio de cuatro ciencias: lógica, ética, metafísica y física, incluyendo en la ética la política y la economía y en física toda la gama de las ciencias naturales programadas en torno a las obras pertenecientes al corpus aristotélico. El profesor ("reader" o "lecturer", palabras intercambiables) hacía su

\footnotetext{
Castro Sánchez, Fernando de Jesús. Estudio Monográfico "La universidad y su aporte al desarrollo tecno científico de la sociedad”. Diciembre 2006. Matanzas. Cuba.

Franco García, José María. La Universidad de Cambridge. Página 1.Universidad de Vigo. España.

Franco García, José María. Op. Cit. Página 1.

Franco García, José María. Op. Cit. Página 2.
} 
exégesis y suministraba una serie inagotable de "questions", que le permitían ser original. $\mathrm{Si}$ el estudiante quería profundizar tenía que hacerlo por su cuenta en la soledad de la tutoría, personalizada y rigurosa, que a la postre sería la que llevó a crear una formación más profunda, que con el tiempo se fue imponiendo y condujo a que Oxford y Cambridge, desde los más remotos tiempos, con Bolonia, París y Salamanca, se impusieran como las grandes universidades de Europa. ${ }^{8}$

¿El secreto de su éxito?.

- Estructura organizacional basada en fundaciones privadas.

- Estabilidad económica y administrativa.

- Integra una sociedad industrial y tecnológicamente desarrollada al servicio de la corona.

- Funciona la cooperación UniversidadEmpresa-Estado.

- Definida política promocional de apoyo incondicional a la educación privada.

- Sus Docentes son Principales (Rector, Decano y Director) y Tutor (profesor dedicados íntegramente a la enseñanza $\mathrm{y}$ formación de un número reducido de alumnos.

- Profesores Investigadores están en los grandes centros de investigación bien equipados, adecuadamente remunerados y con proyectos financiados por el sector privado.

- Existe democracia estudiantil, no se discute legitimidad de la autoridad universitaria. Ausencia de tercio estudiantil en la gestión universitaria.

\section{POSICIONAMIENTO \\ DE UNIVERSIDADES \\ NORTEAMERICANAS.}

El modelo de desarrollo universitario estadounidense parte de una conjunción de los patrones inglés y alemán, pero las características sociales (ante todo económicas) y culturales de este país hacia finales del siglo XIX y durante el siglo XX terminaron imponiendo derroteros propios de desarrollo institucional universitario. ${ }^{9}$

La primera Universidad fundada en territorio norteamericano fue la de Harvard (1636), en una etapa todavía muy temprana del proceso de colonización desarrollado desde Inglaterra a partir de los primeros movimientos de emigrantes. El formato de esta Universidad respondió, por supuesto, al "Residence College" inglés y se concentraba en la actividad de formación para pre graduado procedente de las clases y grupos de mejor posición. ${ }^{10}$

Desde sus inicios ha sido una de las mejores escuelas del mundo. Hoy en día se distingue por su excelencia académica indiscutible en todas las áreas del conocimiento humano y por ser líder en investigación y desarrollo. Harvard tiene la mayor dotación financiera de cualquier organización sin fines de lucro, y la mayor de una institución educativa, aún siendo una universidad privada recibe ayudas del gobierno, instituciones y de sus numerosos ex alumnos. ${ }^{11}$

El sistema de casos prácticos es la base de aprendizaje, sobre todo en su escuela de negocios (Harvard Business School). Se cree que la capacidad tan elevada de análi-

\footnotetext{
8 Franco García, José María. Op. Cit. Página 18-19.

9 Castro Sánchez, Fernando de Jesús. La Universidad y su aporte al desarrollo tecno científico de la sociedad: datos y reflexiones sobre experiencias en Europa, Estados Unidos y Cuba. Pág. 27.

10 Castro Sánchez, Fernando de Jesús. Op. Cit. página 26.

11 http://www.allstudies.com/universidad-de-har
} 
sis y juicio que adquieren sus alumnos es la base de su éxito en la industria y el comercio internacional. Los casos prácticos acercan a los alumnos a situaciones reales de negocios. Con ellos, las clases hacen detonar discusiones sumamente interesantes y proveen lecciones administrativas que los alumnos aprovecharán toda su vida.

La resultante de esta confluencia de factores fue la configuración de un tipo de universidad basada, al igual que la alemana, en una mejor asociación entre docencia e investigación pero, a su vez, con una presencia superior de las ciencias aplicadas y de la actividad ingenieril y tecnológica. En los Estados Unidos, a diferencia de Alemania y de Francia las actividades de ingeniería y tecnologías nunca fueron separadas de las universidades, aún cuando, paralelamente, existieron y se desarrollaron los institutos tecnológicos de nivel superior (Tunnerman, 1990).

Harvard tiene 9 facultades y escuelas ${ }^{12}$ :

- Facultad de Artes y Ciencias con las siguientes Escuelas: Escuela de Ingeniería y Ciencias Aplicada; Escuela de Postgrado de Artes y Ciencias; Escuela de Educación Continua; Escuela de Verano y Escuela de Extensión Universitaria.

- Facultad de Derecho.

- Facultad de Ciencias Empresariales: Escuela de Postgrado de Diseño; Escuela

- Escuela Médica de Harvard.

- Escuela de Odontología de Harvard
Hoy, cuenta con un cuerpo administrativo 14,000 personas, incluyendo una facultad de tiempo completo de 2,000 doctores titulados en el área de su especialidad. Cuarenta premios Nobel y siete presidentes de los Estados Unidos se han graduado en Harvard, además de miles de hombres famosos por su competencia en diferentes industrias.

¿El secreto de su éxito?

- Estabilidad económica y administrativa.

- Eficiente interacción entre UniversidadEmpresa-Estado. Hay impresionante apoyo económico a sus investigaciones.

- Líderes en investigación y desarrollo porque integran una sociedad industrial y tecnológicamente desarrollada.

- Màs de 2,000 Profesores a tiempo completo y todos con título de Doctores.

- Excelentes remuneraciones al reconocido trabajo de sus docentes.

- Preferencia por ciencias aplicadas y actividad ingenieril y tecnológica.

- Sistema de casos es base del aprendizaje.

- Existe democracia estudiantil pero no participan en la gestión universitaria.

- Definida política promocional de apoyo a la educación privada y estatal.

\section{LA UNIVERSIDAD LATINOAMERICANA.}

Entre las Universidades latinoamericanas mejor ubicadas en la lista del "QS World University Rankings", se encuentran las siguientes entidades:

\begin{tabular}{|l|l|l|l|}
\hline INSTITUCIÓN LATINOAMERICANA & UBICACIÒN & ESTATUS & ALUM. \\
\hline Universidad Nacional Autónoma de México & 169 & Estatal & 292,889 \\
\hline Universidad de Sao Paulo & 169 & Estatal & 88,962 \\
\hline Pontificia Universidad Católica de Chile & 250 & Privada & 22,848 \\
\hline Universidad de Chile & 262 & Estatal & 35,619 \\
\hline Universidad de Buenos Aires & 270 & Estatal & 300,000 \\
\hline
\end{tabular}

Fuente: Página Web de cada una de las Universidades; http://es.wikipedia.org

12 http://www.harvard.edu Consultado 15.09.2011. 
Frente a esta realidad nos preguntamos:

- ¿Qué hace la diferencia entre una universidad inglesa y norteamericana con nuestras universidades latinoamericanas?

- ¿Por qué existen distancias abismales en el puntaje obtenido?

Algunas respuestas de profesionales de universidades sudamericanas tratan de explicar esta situación.

1. La directora de la Maestría en Educación de la Universidad de San Andrés e investigadora del Conicet, Silvina Gvirtz, afirmó que "es verdad que América latina no está produciendo conocimiento". Consultada por LA NACION sobre los resultados del informe de The Times, señaló: "Nosotros no tenemos profesores full time, entonces muchos se dedican a la enseñanza y pocos a la investigación. Se debe al vaciamiento sistemático de las universidades producido en la dictadura militar, que se solapó con la política de captación activa por parte de las academias del mundo desarrollado y se siente aún hoy. Además, la investigación no fue una prioridad en la vuelta a la democracia. Hoy hay muchas universidades que son sólo enseñaderos. Tener profesores full time es muy caro y la investigación no tiene repercusiones inmediatas. No requiere sólo políticas y financiamiento por parte del estado sino también una decisión por parte de las universidades, en su criterio de qué priorizar y en qué invertir sus recursos", detalló.

2. Por su parte, la Secretaria de Asuntos Académicos de la Universidad de Buenos Aires, (UBA), Catalina Nosiglia, explicó a LA NACION que "este tipo de rankings no evalúan cosas como la actividad de transferencia y extensión hacia la sociedad, actividades que nuestros estudiantes y docentes realizan mucho. Lo que nosotros producimos de conocimiento y es relevante para nuestras sociedades, no es relevante para ellos". "La UBA tiene muchos desafíos, relacionados con los casi 300 mil alumnos que estudian allí. Uno fundamental es la permanencia y egreso de los alumnos", afirmó Nosiglia. "Los problemas de una universidad de 4000 alumnos y donde ingresan estudiantes de elite, como la de San Pablo, son muy distintos a los que pueda tener la UBA, cuyo objetivo es que cada vez más gente pueda acceder a la universidad", sostuvo.

Las deficiencias que presentan muchas de nuestras universidades y sistemas de ciencia y tecnología se resumen en lo siguiente:

a) Escaso porcentaje de profesores doctores (menos del $20 \%$ en la región)

b) Mínima producción en ciencia y tecnología e innovación

c) Insuficiente porcentaje de investigadores

d) Bajo número de programas doctorales

e) Pequeño porcentaje de movilidad académica y estudiantil (menos del 1\% de la matricula)

La Declaración Mundial de Paris sobre la Educación Superior (1998) enfatizó que las instituciones de educación superior han de enfocar los esfuerzos de desarrollo a las siguientes prioridades: La calidad, la pertinencia y la cooperación internacional. Las Universidades debemos enfocarnos a aspectos importantes tales como:

- Calidad, evaluación y acreditación de los procesos

- Mejoramiento de la planta docente

- Calidad y difusión de la investigación

- Formación integral de los estudiantes

- Transparencia y rendición de cuentas 
El objetivo clave es precisamente que a través de esta cooperación, el intercambio entre las IES (Instituciones de Educación Superior), sea un efecto positivo que contribuya a un mejor desempeño y desarrollo de las mismas, tomando en consideración que esta cooperación coadyuve a la calidad y pertinencia de las IES, en este sentido coincidimos con lo que Jesús Sebastián ${ }^{13}$ menciona, que "el fundamento actual de la cooperación internacional de las universidades, se basa en la complementariedad de sus capacidades para la realización de actividades conjuntas y en la asociación para el beneficio mutuo, esta complementariedad permite la existencia de sinergias y de valores añadidos, constituye la base de la cooperación y revaloriza su calidad".

Analizaremos a continuación cinco áreas fundamentales que hacen la diferencia entre nuestras universidades latinoamericanas calificadas: Estructura Física, Organizaciòn, Población, Administración e Investigación.

\section{UNIVERSIDAD AUTÓNOMA DE MÉXICO. (UNAM). (PUESTO ${ }^{\circ}$ 269)}

¿El secreto de su éxito ${ }^{14}$

- Apoyo político estatal definido al desarrollo de la universidad pública.

- Junta de Gobierno integrada por 15 personas con cargos honoríficos.

- Estructura organizacional es menos complicada que de San Marcos. El Consejo Universitario sólo cuenta con 102 miembros, lo que facilita la toma de decisiones. No existe asamblea universitaria. Junta de Gobierno tiene el voto dirimente.
- Forma parte de una sociedad en proceso de desarrollo industrial y tecnológico superior a los países sudamericanos excepto Argentina y Brasil.

- Excelente interacción entre Universidad-Empresa-Estado.

- $33 \%$ de sus Docentes son a tiempo completo.

- Impresionante estructura física para investigación y desarrollo.

- Ligera ventaja hacia las ciencias exactas que a las ciencias sociales.

- Notable desarrollo en tecnologías de información y comunicación.

- Recibe 1,500 millones de dólares del gobierno mexicano en forma anual.

\section{UNIVERSIDAD DE SAO PAULO (USP). (PUESTO N 269).}

Entre las universidades públicas es la que tiene el mayor número de puestos para carreras de grado y postgrado en Brasil, (en Ciencias Biológicas, Ciencias Exactas y Humanidades), siendo responsable también de la formación del mayor número de Maestros y Doctores en Brasil.

¿El secreto de su éxito?

- Estabilidad económica y administrativa.

- Apoyo político estatal definido al desarrollo de la universidad pública.

- Estructura organizacional simple que prioriza los vicerrectorados con énfasis en investigaciones.

- Preferencia por las Ciencias Exactas sobre Humanidades y Biología.

- Integra una sociedad más desarrollada industrial y tecnológicamente.

13 Doctor en Ciencias Biológicas por la Universidad Complutense de Madrid (1969). Ha sido investigador científico en las Universidades de Wisconsin (1970-71) y Brandeis (1971-73) y en el Instituto de Enzimología y Patología Molecular del que fue vicedirector (1974-83). Vicepresidente de política científica del Consejo Superior de Investigaciones Científicas (CSIC) de España

14 Ley Orgánica de Universidad Nacional Autónoma de México. Publicado 06.01.1945. 
- El 85\% de sus docentes son a dedicación exclusiva y prácticamente el 100\% tienen grado de Doctor.

- Nivel económico aceptable de sus docentes.

- Excelente interacción entre Universidad-Empresa-Estado.

- Buena infraestructura física.

- No existe cogobierno con tercio estudiantil.

\section{PONTIFICIA UNIVERSIDAD \\ CATÓLICA DE CHILE. (PUC). PUESTO 250.}

¿El secreto de su éxito?

- Estabilidad económica y administrativa.

- Estructura organizacional basada en fundaciones privadas. Es simple y prioriza los vicerrectorados con énfasis en investigaciones.

- El $61 \%$ de su Docentes son a tiempo completo y el $92 \%$ tienen grado de Doctor.

- Nivel económico aceptable de sus docentes.

- Excelente interacción entre Universidad-Empresa-Estado.

- Buena infraestructura física.

- Estudiantes tienen participación en el Gobierno pero no alcanzan al tercio estudiantil.

\section{UNIVERSIDAD DE CHILE. (UCH).} PUESTO N .262.

Su principal actividad está focalizada en la presencia de sus cuatro institutos de Investigación:

- Instituto de Estudios Internacionales de la Universidad de Chile, fundada en 1966.
- Instituto de Nutrición y Tecnología en Alimentos de la Universidad de Chile, que funciona desde 1976.

- Instituto de Asuntos Públicos de la Universidad de Chile desde el año 2001.

- Instituto de Comunicación e Imagen de la Universidad de Chile inaugurada en el año 2003. ${ }^{15}$

¿El Secreto de su Éxito?

- Estructura organizacional similar a San Marcos, pero con la tercera parte de personas que facilitan la toma de decisiones. Hay participación estudiantil pero no tienen el peso de un tercio.

- Estabilidad económica y administrativa recién a partir de 2006.

- Buena infraestructura física.

- Investigación focalizada en cuatro Institutos.

- Apoyo político estatal definido al desarrollo de la universidad pública.

- Excelente interacción entre Universidad-Empresa-Estado.

- Nivel económico aceptable de sus docentes.

- Mejor calidad de docentes a tiempo completo y con grado de Doctor

\section{UNIVERSIDAD DE BUENOS AIRES (UBA). ARGENTINA. PUESTO 270.}

Cerca del 30\% de la investigación científica del país se realiza en esta institución. El $31.3 \%$ del personal docente censado ha declarado participar en proyectos de investigación acreditados $\mathrm{y} / \mathrm{o}$ financiados por UBA, $\mathrm{u}$ otras instituciones. De los 9,048 investigadores, el $76 \%$ declaró realizar sus actividades de investigación en el ámbito de la Universidad.

15 http://es.wikipedia.org/wiki/Universidad_Nacional_Aut\%C3\%B3noma_de_M\%C3\%A9xico 
¿El Secreto de su Éxito?

- Estructura organizacional menos complicada que San Marcos. Menos integrantes y màs rápida toma de decisiones. No tiene Asamblea Universitaria.

- Estabilidad económica y administrativa recién a partir de 2006.

- Apoyo económico anual del Estado de 165 millones de dólares.

- Forma parte de una sociedad con elevado desarrollo industrial y tecnológico.

- Notablemente focalizada en la investigación científica.

- Sólo tiene Tercio Estudiantil a nivel del Consejo de Facultad.

- Nivel económico aceptable de sus docentes.

\section{UNA PROPUESTA A LA REAL SITUACIÓN DE LA UNIVERSIDAD PÚBLICA: UNMSM}

"La reestructuración apunta a lograr una mayor eficiencia en el servicio que damos a nuestros usuarios, de tal manera que todos los estamentos resulten beneficiados", sostuvo nuestro flamante Rector Dr. Pedro Cotillo Zegarra.

¿Cómo alcanzar esta mayor eficiencia en nuestra universidad? Analicemos las fortalezas de las universidades líderes del primer mundo:

1. Organigrama estructural simplificado.

2. Órganos de gobierno con menor número de miembros.

3. Orden y respeto a la autoridad constituida.

4. Mayor presencia de profesores a tiempo completo.

5. Obligatoriedad del grado de Doctor para ser Docente.
6. Apoyo total a la investigación aplicada al servicio de la empresa y el estado.

¿Qué nos ofrece el proyecto de la nueva Ley universitaria en este orden cosas?

- Mejorar organización académica en Facultades, Escuelas Profesionales, Departamentos Académicos, Unidades de Postgrado e Institutos de Docencia e Investigación. (Artículo 13. Incisos a, b, c, d.)

$\mathrm{Al}$ respecto los Institutos de Investigación, pueden mejorar su rendimiento si se fusionan en Cinco Sectores de suerte que aparezcan cinco unidades en vez de 20:

a) Institutos de Investigación en Ciencias Básicas: Biología, Física y Matemáticas.

b) Instituto de Investigación en Ciencias de la Salud: Farmacia y Bioquímica, Medicina Humana, Medicina Veterinaria, Odontología, Psicología.

c) Instituto de Investigación en Ingenierías: Geología, Minas, Ciencias Metalúrgicas y Ciencias Geográficas; Química e Ingeniería Química, Ingeniería Electrónica, Ingeniería de Sistemas, Ingeniería Industrial.

d) Instituto de Investigación en Ciencias Empresariales: Ciencias Administrativas, Ciencias Contables y Ciencias Económicas, Derecho y Ciencias Políticas.

e) Instituto de Investigación en $\mathbf{H u}$ manidades: Educación, Letras y Ciencias Humanas, Ciencias Sociales.

¿Qué antecedente tenemos al respecto? El caso de la Universidad de Chile que 
tiene 35,619 alumnos, administra cuatro (4) Institutos, es responsable del $37 \%$ de las investigaciones científicas chilenas y está ubicado en el puesto 262 del reciente Ranking de Universidades. Esta integración de Institutos, facilita además, la investigación multidisciplinaria, y alianzas estratégicas con entidades públicas y privadas, como académico-profesional al interior de la Universidad. Y facilitará definir el 5\% de Investigadores a Dedicación Exclusiva que el proyecta fija un plazo de 5 años (artículo 19).

- Fusionar la estructura organizacional de las Unidades de Postgrado, que en vez de 20, siguiendo el mismo procedimiento anterior, formarían cinco (5) unidades integradas, como administra su Postgrado, la universidad más grande de América Latina: la Universidad Autónoma de México.

- Fusionar la estructura organizacional de los Centros de Extensión Universitaria y Proyección Social, que en vez de 20, siguiendo el mismo proceso anterior, formarían cinco (5), unidades integradas.

- Los Centros de Producción de la Universidad, según el artículo 44 del Proyecto de Ley, pasan al ámbito del Fondo Nacional de la Actividad Empresarial del Estado (FONAFE).

- Sobre el gobierno de la Universidad, según el artículo 24 del Proyecto, se mantiene la misma estructura vigente: Asamblea Universitaria, Consejo Universitario, Rector, Consejos de Facultad y Decanos. Al respecto, el problema de gestión de nuestra entidad, es el entrampamiento para la toma de decisiones.

- En este aspecto lo ideal sería contar solamente con un Consejo Universitario con
9 miembros como propone el artículo 27 del Proyecto de Ley.

¿Qué antecedentes tenemos al respecto?. El caso de la Universidad de Buenos Aires, que luego de una crisis institucional en el año 2006, el Consejo Superior presidido por el Rector convocó a alecciones directas, secretas y universales. Como ahora propone el Proyecto de Ley en su artículo 30 y siguientes.

\section{CONCLUSIONES}

a) Las universidades inglesas por ser las más antiguas del mundo basan el éxito de su desarrollo en la estabilidad económica institucional, dedicación a la ciencia experimental $y$, fundamentalmente, a la instauración de la "tutoría", donde el profesor dedica su tiempo íntegramente a la enseñanza y formación de un nùmero selecto, exclusivo y reducido de alumnos, con remuneración suficiente que solucionan problemas que agobian su diario vivir. $\mathrm{Y}$ en el aspecto organizacional funcionan como empresas privadas con eficiencia, eficacia y efectividad, formando líderes para la sociedad al servicio de la corona y de la empresa privada, base del capitalismo.

b) Las universidades norteamericanas, inicialmente partieron del modelo inglés, pero en otro tiempo y lugar, se integraron al modelo alemán cuyo énfasis era la investigación científica en base a datos cuantitativos y la experimentación. Pero por circunstancias históricas con ocasión de la segunda guerra mundial (19391945), enfrentado el país a la urgencia de reconstruir Europa y Japón, devastados por esta conflagración, marcado por el interés de satisfacer necesidades mun- 
diales, sus universidades, industrias y el poder de ser naciente potencia mundial, volcaron sus experimentos a la solución de las necesidades del mercado. Constancia de esta afirmación es el "modelo de casos" base de aprendizaje que popularizó la Universidad de Harvard en el mundo. Sus instituciones universitarias, tanto públicas como privadas, al igual que las británicas, funcionan como empresas con orden, eficiencia y efectividad. Mención especial es la obligación de dedicación exclusiva y grado de Doctor para todos los profesores que trabajan en estas organizaciones.

c) La universidad latinoamericana tiene otro origen y hoy, merced al estudio bajo análisis, notamos en el tiempo diferentes resultados en función del énfasis por las ciencias exactas o las ciencias sociales que cada país ha preferido. Estas instituciones llegaron al continente "trasplantadas" como un producto experimentado de las Universidades de Salamanca y de Alcalá de Henares. Excepto Brasil, que recién en 1934 funda sus primeras universidades. Por tanto no heredó el famoso "Grito de Córdoba" que introdujo en la gestión de nuestras universidades públicas el cogobierno màs conocido como el tercio estudiantil. La exploración de las universidades analizadas muestra una realidad: son más competitivas aquellas que orientan sus estudios a las ciencias exactas, como las universidades brasileñas y priorizan su capital humano mediante permanente capacitación externa.

\section{REFERENCIAS BIBLIOGRÁFICAS}

1. Arosena Rodrigo \& Sutz, Judith. La Universidad Latinoamericana del Futuro.
Tendencias. Escenarios. Alternativas. Revista Ciencias, Tecnología, Sociedad e Innovación. Organización de Estados Iberoamericanos.

2. Castro Sánchez, Fernando de Jesús. La Universidad y su aporte al desarrollo tecno científico de la sociedad: Datos y Reflexiones sobre experiencias en Europa, Estados Unidos y Cuba. Ciudad de Matanzas. Diciembre 2006. Cuba.

3. Franco García. José María. La Universidad de Cambridge. Ensayo.

4. Giraldo Reyes, Beatriz Helena. Desarrollo del Concepto de Investigación en la Educación Superior para América Latina. Universidad Javeriana. Cali. Colombia.

5. Montoro Sánchez, Maria Ángeles. Mora Valentín, Eva María. Hacia una Gestión Eficaz de las Relaciones entre empresas y Universidades. Universia Business Review. Segundo Trimestre 2006. España.

6. Tunnermann Bernheim, Carlos. La Educación Permanente y su Impacto en la Educaciòn Superior. Documentos sobre Educaciòn Superior. UNESCO. 1990.

\section{ELECTRÓNICAS}

1. http://www.topuniversities.com/university-rankings/world-university-rankings/2011 Consultado 15.09.2011

2. http://www.allstudies.com/universidad-de-harvard.html. Consultado 10.09.2011.

3. http://www.harvard.edu Consultado 15.09.2011.

4. http://es.wikipedia.org/wiki/Universidad_Nacional_Aut\%C3\%B3noma_ 
de_M\%C3\%A9xico.Consultado 11.09.2011.

5. http://www.usp.br/internacional/ home.php?id_cont=28\&idioma=es Consultado 13.09.2011.

6. http://es.wikipedia.org/wiki/Universidad_de_S\%C3\%A3o_Paulo. Consultado 13.09.2011

7. http://www.usp.br/internacional/ home.php?id_cont $=28$ \&idioma $=$ es Consultado 13.09.2011.

8. http://www.wikipedia.org/wiki/Pontificia_Universidad_Cat\%C3\%B3lica_ de_Chile 07.09.2011.
9. http://www.uc.cl/webpuc/site/launiversidad/uccifras.html. Consultado 13.09.2011.

10. www.uchile.cl consultado 13.09.2011

11. http://es.wikipedia.org/wiki/Universidad_de_Chile.Consultado 15.09.2011.

12. Rankings.americaeconomia.com/2010/ mejores-universidades-chile/calidad_ docentes.php. Consultado el 15.09.2011.

13. www.uchile.cl consultado el 15.09.2011. 14. http://es.wikipedia.org/wiki/Universidad_de_Buenos_Aires . Consultada 13.09.11 